\title{
Electronic Medical Records in Colorectal Surgery
}

\author{
Matthias Turina, MD, $\mathrm{PhD}^{1}$ Ravi P. Kiran, MBBS, $\mathrm{MS}^{1}$ \\ ${ }^{1}$ Department of Colorectal Surgery, Cleveland Clinic Foundation, \\ Cleveland, Ohio \\ Address for correspondence Ravi P. Kiran, MBBS, Department of \\ Colorectal Surgery, Cleveland Clinic Foundation, 9500 Euclid Ave., \\ A30, Cleveland, OH 44195 (e-mail: kiranp@ccf.org).
}

Clin Colon Rectal Surg 2013;26:17-22.

Abstract
Keywords
- electronic medical
record
- protected health
information
- data
- colorectal
- colon
- surgery
- HIPAA
- billing

Electronic medical records (EMRs) are being widely implemented today, either as standalone applications in smaller practices or as systems-based integrated network solutions in larger health care organizations. Advantages include rapid accessibility, worldwide availability, ease of storage, and secure transfer of protected health information (PHI). Computerized physician order entry (CPOE) and decision-support capabilities such as the triggering of an alarm when multiple medications with known interactions are ordered, as well as the seemingly endless possibilities for electronic integration and extraction of PHI for clinical and research purposes, have created opportunities and pitfalls alike. Risks include breaches of confidentiality with a need to implement tighter measures for electronic security. These measures contrast efforts required for the realization of common data formats that have national and even international compatibility. EMRs provide a common platform that could potentially allow for the integration and administration of clinical care, research, and quality metrics, thus promoting optimal outcomes for patients. Technical and medicolegal difficulties need to be overcome in the years to come so that the safe use of PHI can be ensured while still maintaining the benefits and convenience of modern EMR systems.
Objectives: On completion of this article, the reader should be able to summarize current developments in the implementation of electronic medical records in the United States, and name challenges related to patient confidentiality by the use of such systems.

An electronic medical record (EMR, syn. EHR, electronic health record) is a computerized medical record that allows ubiquitous storage, retrieval, and analysis of patient data from a hospital or physician's office. EMRs may be a part of a local stand-alone health information system or be integrated into a network of health care providers such as a group of hospitals along with affiliated regional physician offices. Even though EMR systems with computerized (physician) order entry (C $[\mathrm{P}] \mathrm{OE}$ ) have existed for more than 30 years, considerable time passed before the widespread adoption of such systems occurred in the United States. In fact, fewer than $10 \%$ of U.S. physicians had implemented a basic functional EMR system as defined by the Robert Wood Johnson Foundation ${ }^{1}$ before 2006 , but the adoption rate rose quickly thereafter. ${ }^{2}$ In 2010 ,
$24.9 \%$ of providers would use such a system and roughly $50 \%$ of all physician offices would be using an EMR system of any sort, trend rising (-Fig. 1). From a nationwide perspective as documented by the Centers for Disease Control (CDC) (-Fig. 2), EMR systems appear to be adopted more quickly in northern as opposed to southern states, even though this does not reflect progress made by individual health organizations. $^{2}$

The following article is meant to provide an overview of EMR technology and its uses in clinical care and research in 2012. Special emphasis has been placed on its use in colorectal surgery; however, most aspects described in this text equally pertain to other medical and surgical subspecialties. We also provide an example of how clinical care and research have been integrated in a busy colorectal unit.

\section{Extracting and Integrating Data}

Rapid, reliable, and uncomplicated access to protected patient health information (PHI) is crucial to modern health care and has been the focus of public attention in recent years. In 2009,
Issue Theme Technology and Health Information in Colorectal Surgery; Guest Editor, Scott R. Steele, MD.
Copyright $\odot 2013$ by Thieme Medical Publishers, Inc., 333 Seventh Avenue, New York, NY 10001, USA. Tel: +1(212) 584-4662.
DOI http://dx.doi.org/ 10.1055/s-0033-1333629. ISSN 1531-0043. 


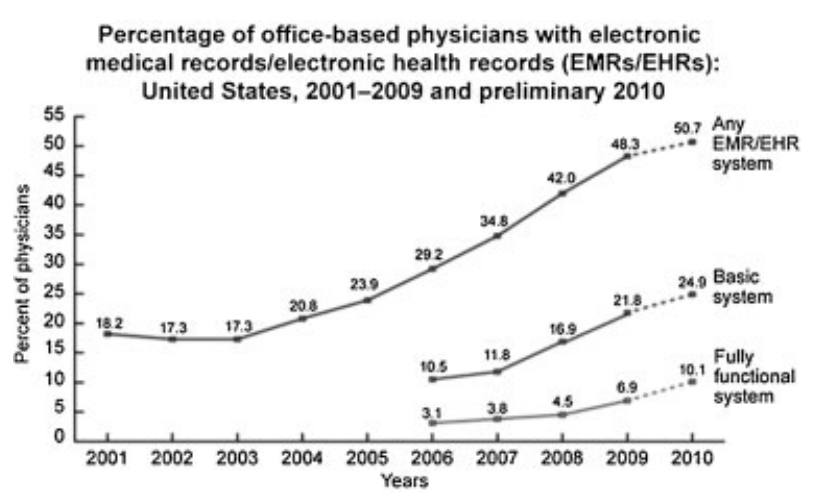

Fig. 1 Any electronic medical record (EMR) or electronic health record (EHR) is an electronic or health medical record that is either all or partially electronic (excluding systems solely for billing). The 2010 data are preliminary estimates (as shown by dashed lines). Estimates of basic and fully functional systems prior to 2006 could not be computed because some items were not collected in the survey. Fully functional systems are a subset of basic systems and include advanced features such as drug interaction or contraindication warning, or electronic transfer of prescriptions to pharmacies. Nonfederal, office-based physicians are included; radiologists, anesthesiologists, and pathologists are excluded. Source: CDC/NCHS, National Ambulatory Care Medical Survey.

the federal government passed the Health Information Technology for Economic and Clinical Health (HITECH) act as part of the American Recovery and Reinvestment Act, also known as the stimulus bill. ${ }^{3}$ The goal of HITECH is to establish a nationwide, interoperable, and secure health information system by financially promoting the use of electronic health or medical record (EMR) systems by individual providers. In fact, the federal government authorized grants and incentives totaling an estimated $\$ 14$ billion to $\$ 27$ billion to promote "meaningful use" of EMRs by providers. ${ }^{4}$ With the definition of "meaningful" left purposefully vague, the intended consequence (and ongoing result) was to spur all types of practicefrom independent providers to large health care systemsinto migrating toward EMR use.

By the maintenance of a comprehensive record of patient health, disease, and response to treatment, EMRs facilitate the use and availability of patient data for treatment, billing, storage, and hence, retrieval. By design, EMRs further lend themselves well for clinical, epidemiologic and informatics research, provided methods for specific and inclusive extraction of data are set in place. Clinical data, however, are mostly available in narrative form as transcription of dictations, direct entry by providers, or use of speech recognition applications. This free-text form is convenient for use in day-to-day clinical work, but its structure is difficult for searching, summarization, decision-support, or statistical analysis. Automated approaches, such as natural language processing (NLP), that extract specific medical information from textual documents without the use of discharge codes offer a powerful alternative to unreliable and incomprehensive administrative data, or labor-intensive, expensive manual chart reviews. ${ }^{5}$ In fact, the feasibility and clinical benefit of NLP-driven algorithms has been shown in several studies ${ }^{6-8}$ : $\mathrm{Xu}$ et al were able to demonstrate an algorithm combining machine learning and NLP to identify patients with colorectal cancer from entire EMRs at Vanderbilt University Hospitals using both various free-text chart notes and coded data such as ICD-9 and CPT codes. ${ }^{8}$ Similarly, in an attempt to facilitate identification of patients with correct timing and completion of screening colonoscopy, Denny et al were able to demonstrate the superiority of NLP over billing codes with a $95 \%$

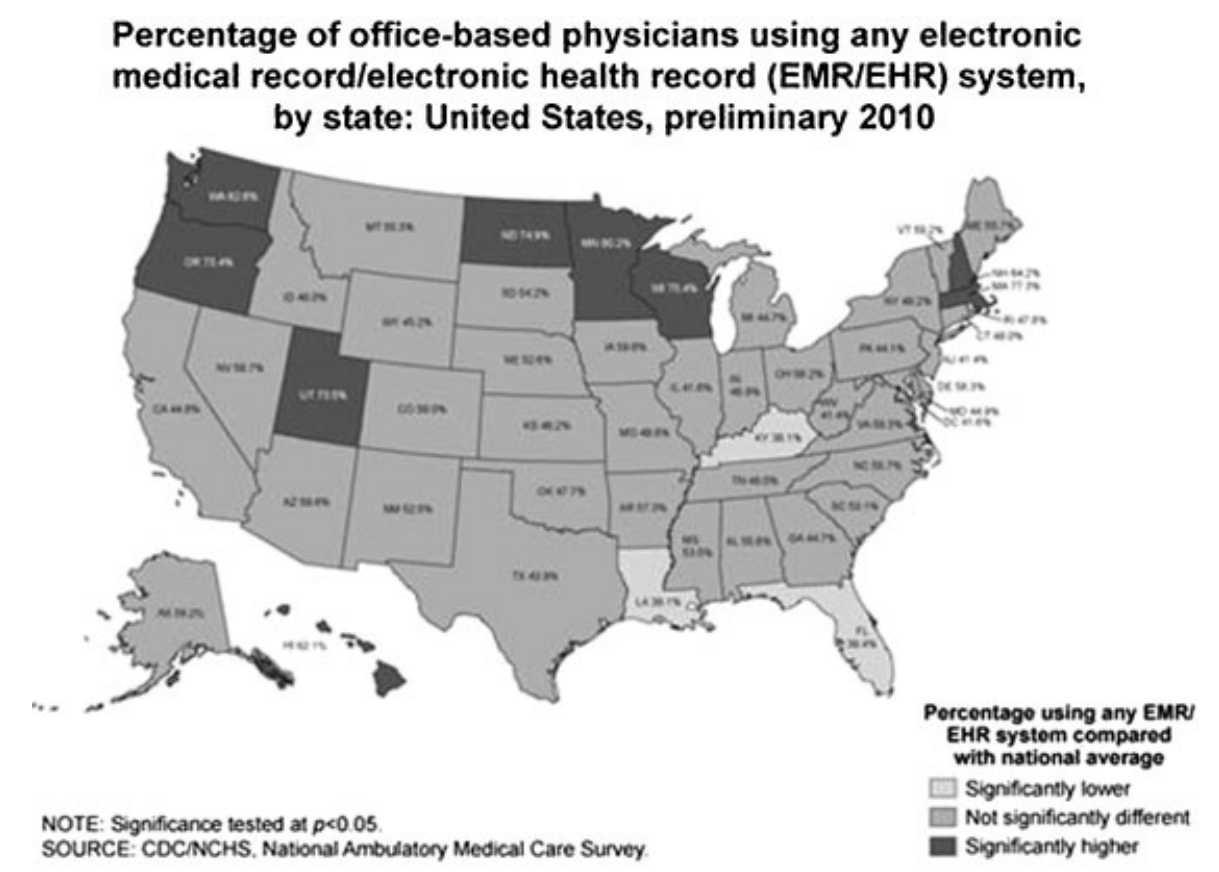

Fig. 2 Source: CDC/NCHS, National Ambulatory Care Medical Survey. CDC, Centers for Disease Control and Prevention; NCHS, National Center for Health Statistics. 
precision compared with the gold standard of manual EMR review by expert physicians. ${ }^{7}$

Finally, Murff et al examined the sensitivity and specificity of NLP analysis to detect postoperative complications on 2,974 patients from six Veterans Administration (VA) hospitals in comparison to patient safety indicators that use discharge information. ${ }^{6}$ In their study, NLP correctly identified $82 \%$ of acute renal failure events, $59 \%$ of venous thromboembolism, $64 \%$ of pneumonia, $89 \%$ of sepsis and $91 \%$ of myocardial infarction events at a high specificity, all of which surpassed patient safety indicators based on administrative coding. Although there remains room for improvement, these results underline the capabilities of NLP-driven analyses. On the downside, there is definitely a learning curve from the perspective of the physician to both read and chart a typical patient encounter with some of these systems. As the patient's condition becomes more complex, often the software is unable to express the various aspects of their disease process and care. Although "free-text" abilities were initially meant for use in these specific instances, they often become the primary method of charting, resulting in a degradation of the wide variety of potential uses.

As laudable as the use of such refined EMR technology by single-provider organizations undoubtedly may be, it also raises the question of systems compatibility between different institutions or states. To this year, great progress has already been achieved in adopting EMRs in individual hospitals and provider systems, yet the integration of such systems into a national or even international standard is not likely to occur in the near future: Different security systems, coding variability, and multiple types of EMR systems result in an inability to communicate with each other. Despite the obvious complexity of such an endeavor, the lack of progress is especially obvious when comparing the medical to the financial industry: The latter has created a world in which we can access our personal monetary information and withdraw local currency from our home account at the click of a button. Yet at the same time, when we are referred from a primary care physician to a specialist's office in our hometown (even within the same system), we need to start from zero in providing all personal health information again, mostly in paper format. ${ }^{9}$ This is further compounded when referrals or transfers occur between health care organizations, with essentially reliance on direct communication or a paper chart that may or may not have been copied in total. Financial institutions have mastered the secure integration, rapid availability, and international transfer of protected information in real-time, while medicine has obviously lagged behind. However, several initiatives have been set forth to create international standards or a common data language, which will be described in more detail below.

\section{Use in Outcomes Research}

Prior to the introduction of EMRs, the best source of data for clinical research was claims data. The big disadvantage of claims data, however, is that it contains only demographics, diagnoses, and procedures relevant for billing purposes that are not necessarily reflective of the individual patient's medical condition. This is where EMRs exhibit their full potential, as data capture and integration into predefined databases allow convenient and comprehensive analysis of any factor in the original EMR dataset.

From an information technology (IT) standpoint, however, the use of a stand-alone EMR system for outcomes research is just a beginning. Provided adequate IT capabilities and support are available, inclusion of patient-specific data from associated providers and institutions such as anesthesiology, pathology, or even social security data are feasible, and allows for the comprehensive integration of all essential aspects of the individual patient's health care. - Fig. $\mathbf{3}$ is an illustration of how EMR data are being merged with PHI from various sources at the authors' institution. This allows for the creation of a comprehensive database that collates information of all patients undergoing surgical treatment in the Department of Colorectal Surgery at the Cleveland Clinic. The main advantage of such an integrative outcomes database is the potential for inclusion of PHI for large numbers of patients with a minimal need for manual entry. In addition to the obvious advantages of efficiency, the process increases reliability and reproducibility of the data obtained for future analyses.

The traditional processes of integrating patient data into EMRs by patient interview and incorporation of external provider data can be further enhanced by integrating patient-reported outcomes into the workflow. In a pilot study at the Department of Colorectal Surgery at the Cleveland Clinic, tablet computing technology was used to obtain patientreported outcomes from 103 office visits to two surgeons while patients were still in the waiting area of the outpatient clinic. ${ }^{10}$ When patients checked in at least 30 minutes early, the questionnaire response rate was 96\%, which compares very favorably to a historic internal control using mailed questionnaires in which the response rate was a mere 52\%. In addition, the questionnaires were automatically scored and their data seamlessly integrated into the patients EMR upon provider approval, thereby greatly reducing the amount of human intervention required for data acquisition. Finally, patient satisfaction with the electronic format was gratifyingly high, with patients strongly preferring the tablet-based over traditional mailing questionnaires.

However, acquisition and transfer of PHI by EMRs are being closely scrutinized for potential breaches of confidentiality, which is especially relevant in outcomes research due to the risk for harm to many subjects and the large amount of data processed.

In the United States, the HIPAA (Health Insurance Portability and Accountability Act, codified as 45 CFR $\$ 160$ and 164) protects the confidentiality of patient data, whereas the Common Rule (codified as 45 CFR §46) protects the confidentiality of research subjects. In essence, these regulations require an informed consent by the patient to conduct analysis of protected patient health information and previous approval of the Institutional Review Board (IRB). Informed consent may be waived if patient data are de-identified according to a list of established patient identifiers. Noncompliance of these protective measures resulting in a breach of 


\section{Colorectal Surgery Outcomes Registry}

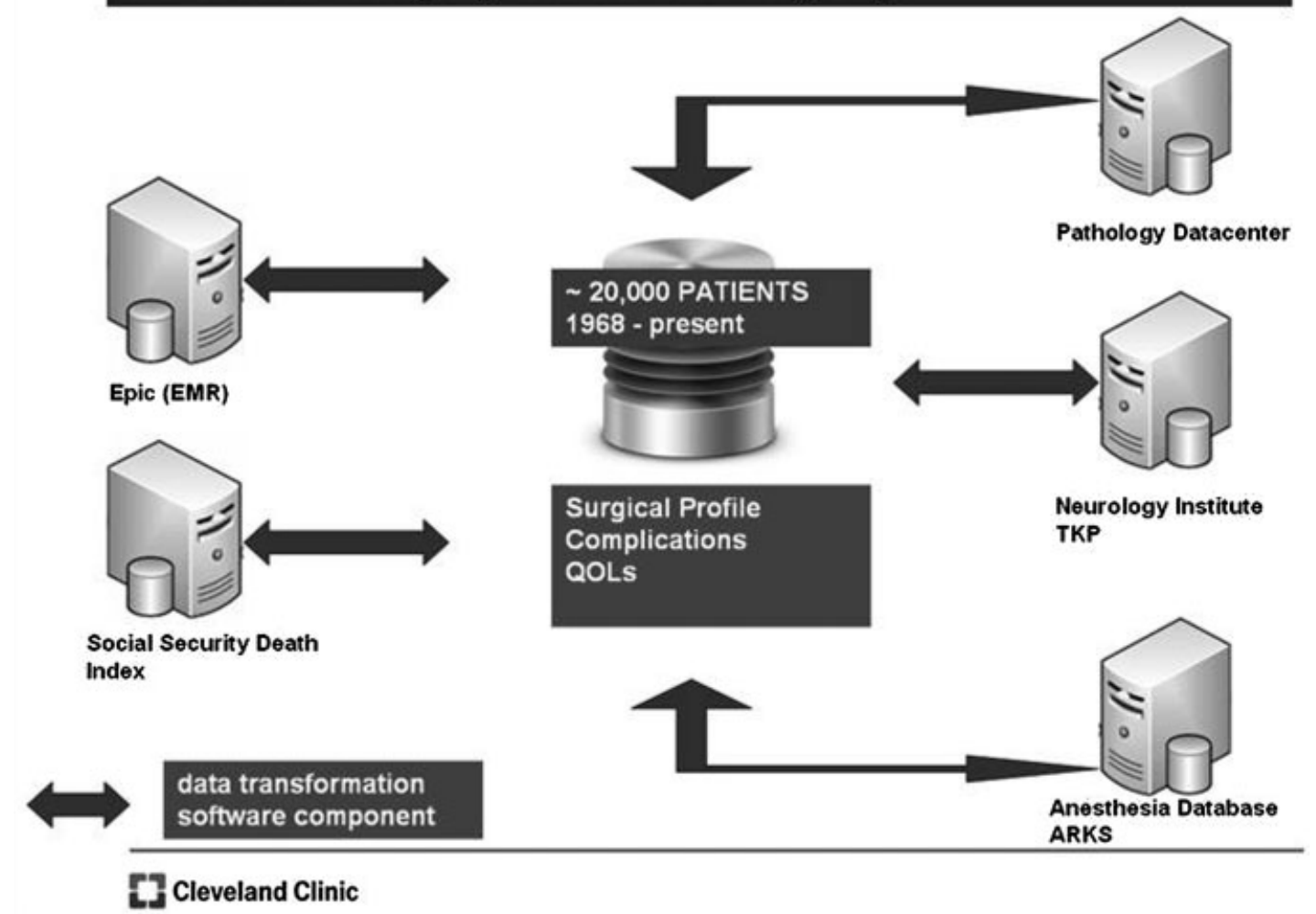

Fig. 3 Layout of the Outcomes Database at the Cleveland Clinic Department of Colorectal Surgery. The interconnectivity between the database, the original EPIC (@ 2012 Epic Systems Corporation) electronic medical record (EMR) source, and data from other sources is shown. QOL, quality of life; TKP, the Knowledge program (a program that allows integration of patient self-reported outcomes into the EMR); ARKS, anesthesia record keeping system.

confidentiality can have substantial consequences for the involved institutions. Only recently, a California medical center faced a six-digit fine following a self-reported breach of confidentiality in one trauma patient's EMR, which was recognized in an internal audit. ${ }^{11}$ Similar occurrences have left health care providers confused and frustrated about how to combine the obvious advantages of EMRs without forsaking patient privacy. In essence, both health care providers and medical researchers face a "catch-22" situation by implementing EMRs that are widely promoted for their ease of sharing data, while making it extremely difficult to ensure security of individual patients protected health information.

\section{The Impact of the EMR}

EMR technology has the additional inbuilt ability for computerized physician order entry and decision support capabilities. ${ }^{12}$ Thus, the system could be configured to trigger an alarm when an order is placed that results in an interaction with an existing medication. ${ }^{13}$ In 1999, Bates et al showed that computerized POE with the addition of decision support features reduced medication errors other than missed doses by $81 \%{ }^{14}$ Decision support works through the application of defined rules for structured data such as laboratory test results or a list of active medications, which is what comput- ing is all about-the storage and running of algorithms on structured data. ${ }^{15}$ This is just one example of how even basic EMR systems can facilitate individual health care and improve patient safety. Similarly, triggers may be set within order-sets that promote the adoption of predefined clinical care pathways and alert users of potential deviations from recommended guidelines. For example, such triggers could easily be set to foster the universal adoption of and minimize deviations from Surgical Care Improvement Project (SCIP) guidelines.

Another great advantage of EMR systems is the potential for electronic linkages to external provider systems. Modern EMR systems allow email communication and secure messaging among health care providers or even between providers and patients within the same network or organization. Such direct communication may be used to inform patients about results from recent tests or to administer requests such as a prescription refill. ${ }^{16,17}$ Although such network applications are currently rare or still in the development phase, ${ }^{18}$ their implementation is likely to increase not only due to the federal government's recent financial commitment, but more simply also due to their technical feasibility of the approach and ability to greatly facilitate the providers' work. ${ }^{19}$

A requirement for any larger regional, national, or even international network is the existence of a common data 
format or language to enable interoperability of the information-system. Such initiatives are currently underway and have already defined global, platform-independent data standards by which to enable acquisition, transfer, and analysis of protected health information. ${ }^{9}$ Notably, Health Level Seven (HL7), a standards-development organization accredited by the American National Standards Institute and located in Ann Arbor, Michigan, has defined standards for the transfer of protected patient health information during the past two decades. In parallel, the Clinical Data Interchange Standards Consortium (CDISC) has devised similar standards for clinical research data. In 2001, HL7 and CDISC found a way to synchronize their individual standards to create a platform on which both systems (i.e., those supporting healthcare applications and those used in clinical research) will essentially speak the same language. ${ }^{9}$ Due to the number of systems currently in use, this may not be feasible in the near future; however, mandates for unified basic formatting may allow the continuation of separate EMR systems while providing interchangeable secure data access.

\section{Billing, Coding, and Reimbursement to Enhance a Practice}

Today's health care system is a business in which it has become increasingly difficult for individual providers to remain profitable. Reimbursement is based on correct coding, which relies on accurate and comprehensive discharge summaries. Delays in the completion of discharge summaries may result in billing delays and deferred payment, which may reach tens of thousands of dollars in complex surgical cases and put a significant strain on any institution's finances. Early electronic availability of discharge information may simplify and accelerate coding and thereby improve billing efficiency. A recent analysis from the University of California San Francisco by Mourad and colleagues showed that $\sim 20 \%$ of billing is delayed due to incomplete discharge documentation. For a hospital that generates billing revenue exceeding $\$ 1.4$ billion per year, delayed payment may lead to significant financial losses. ${ }^{20}$ In this case the EMR provides a reliable, rapid and reproducible solution, and may more than pay for the initial investment.

Opportunities to maximize reimbursement are frequently missed. The problem of deferred and incomplete reimbursement is by no means restricted to major medical centers. Smaller physician-owned practices are equally susceptible to increasing costs and diminishing revenues, and are often well advised to implement systems that allow the selection of billing codes at the end of each encounter. Heidelbaugh et al found that at a Medicare reimbursement rate of $\$ 96.01$ for a “99214" visit and $\$ 63.73$ for a "99213" visit in 2008, a physician who undercodes just one level 4 visit per day could lose as much as $\$ 8,393$ over the course of a year. ${ }^{21}$

Buying and implementing an EMR system is a costly investment. The financial aspects and pros and cons thus need to be well researched and its introduction into daily practice appropriately planned. With a plethora of software systems on the market that differ widely in their capabilities, it is essential to look past the immediate needs of the clinic and try to anticipate future growth and requirements early. When evaluating the purchase of a de novo EMR system, EMRs with an integrated billing module may provide distinct advantages and financial incentives long term, as they avoid paper-based submission of claims. In addition, the savings in time previously devoted to manual filing and submission of claims are clear advantages that provide a clear incentive to any provider or organization because this would decrease the administrative burden on physicians, letting them do what they are meant to do-treat patients.

\section{Incorporating EMR into Your Practice}

Despite the United States having seemingly lagged behind in the implementation of EMRs in past years, the current momentum to adopt such technology is considerable, and is reflected in President Obama's ambitious goals to provide an EMR to every American by $2014 .^{22}$ Establishment of nationwide network standards and concerns over legal implications regarding security of PHI are slowing this process, but both issues are likely to be resolved in the coming years. When it comes to whether or when to incorporate EMR systems in existing provider organizations, considerable start-up costs and risks must be weighed against the sizeable, but uncertain long-term benefits. ${ }^{23}$ Although the malpractice-related implications of EMRs are important in any discussion that evaluates their risks versus benefits, it may be argued that on balance, the wealth of stored electronic documentation in modern EMRs will provide an advantage to providers when faced with the scrutiny of malpractice claims. In fact, Virapongse et al have shown that physicians using EMRs have a lower number of paid malpractice claims, even though this finding did not withstand multivariate analysis. ${ }^{24}$

Finally, as the use of EMRs grows, failure to adopt such systems may itself represent a deviation from an expected standard of care, which is defined by reference to what is customary among peers. ${ }^{25}$ Undoubtedly, several concerns will likely persist and new problems continue to emerge as EMR systems are implemented across the nation. Particular challenges include the integration of measures that promote system intercompatibility, while simultaneously restricting protected health information to select providers or EMR users. Nevertheless, the worldwide trend toward the adoption and development of more integrative and user-friendly network systems has been obvious long before the 2009 stimulus bill, and is unlikely to be stalled by medicolegal concerns. Once solutions have been found to overcome current technical and legal issues, EMR technology may indeed fulfill its promises and make contemporary health care a more effective, user-friendly, and safe environment for providers and patients alike.

\section{References}

1 The Robert Wood Johnson Foundation. Health information technology in the united states: where we stand, 2008. Available at: http://www.rwjf.org/files/research/062508.hit.exsummary.pdf. Accessed March 21, 2012 
2 Hsiao CJ, Hing E, Socey T, et al. Centers for Disease Control and Prevention: NCHS Health E-Stat. Electronic medical record / electronic health record systems of office-based physicians: United States, 2009 and preliminary 2010 state estimates. Available at: http://www.cdc.gov/nchs/data/hestat/emr_ehr_09/emr_ehr_09. htm. Accessed March 20, 2012

3 Blumenthal D. Stimulating the adoption of health information technology. N Engl J Med 2009;360(15):1477-1479

4 Health Information Technology for Economic and Clinical Health Act of 2009 (HITECH Act). , Pub. L. No. 111-5, Div. A, tit. XIII, Div. B, tit. IV, Feb. 17, 2009, 123 Stat. 226, 467 (codified in scattered sections of 42 U.S.C.A.); 2012

5 Meystre SM, Savova GK, Kipper-Schuler KC, Hurdle JF. Extracting information from textual documents in the electronic health record: a review of recent research. Yearb Med Inform 2008: 128-144

6 Murff HJ, FitzHenry F, Matheny ME, et al. Automated identification of postoperative complications within an electronic medical record using natural language processing. JAMA 2011;306(8):848-855

7 Denny JC, Peterson JF, Choma NN, et al. Extracting timing and status descriptors for colonoscopy testing from electronic medical records. J Am Med Inform Assoc 2010;17(4):383-388

$8 \mathrm{Xu} \mathrm{H}, \mathrm{Fu} \mathrm{Z}$, Shah A, et al. Extracting and integrating data from entire electronic health records for detecting colorectal cancer cases. AMIA Annu Symp Proc 2011;2011:1564-1572

9 Kush RD, Helton E, Rockhold FW, Hardison CD. Electronic health records, medical research, and the Tower of Babel. N Engl J Med 2008;358(16):1738-1740

10 Gurland B, Alves-Ferreira PC, Sobol T, Kiran RP. Using technology to improve data capture and integration of patient-reported outcomes into clinical care: pilot results in a busy colorectal unit. Dis Colon Rectum 2010;53(8):1168-1175

11 Sarrico C, Hauenstein J. Can EHRs and HIEs get along with HIPAA security requirements? Healthc Financ Manage 2011;65(2):86-90

12 Chaudhry B, Wang J, Wu S, et al. Systematic review: impact of health information technology on quality, efficiency, and costs of medical care. Ann Intern Med 2006;144(10):742-752
13 Kaushal R, Shojania KG, Bates DW. Effects of computerized physician order entry and clinical decision support systems on medication safety: a systematic review. Arch Intern Med 2003;163 (12):1409-1416

14 Bates DW, Teich JM, Lee J, et al. The impact of computerized physician order entry on medication error prevention. J Am Med Inform Assoc 1999;6(4):313-321

15 Jha AK. The promise of electronic records: around the corner or down the road? JAMA 2011;306(8):880-881

16 Mangalmurti SS, Murtagh L, Mello MM. Medical malpractice liability in the age of electronic health records. N Engl J Med 2010;363(21):2060-2067

17 Ralston JD, Coleman K, Reid RJ, Handley MR, Larson EB. Patient experience should be part of meaningful-use criteria. Health Aff (Millwood) 2010;29(4):607-613

18 Adler-Milstein J, Bates DW, Jha AKUS. U.S. Regional health information organizations: progress and challenges. Health Aff (Millwood) 2009;28(2):483-492

19 Blumenthal D. Launching HITECH. N Engl J Med 2010;362(5): 382-385

20 Mourad M, Cucina R, Ramanathan R, Vidyarthi AR. Addressing the business of discharge: building a case for an electronic discharge summary. J Hosp Med 2011;6(1):37-42

21 Heidelbaugh JJ, Riley M, Habetler JM. 10 billing \& coding tips to boost your reimbursement. J Fam Pract 2008;57(11):724-730

22 Pear R. New York Times, January 17,2009. Available at: http:// www.nytimes.com/2009/01/18/us/politics/18health.html? _r $=3 \&$ ref=health. Accessed March 19, 2012

23 DesRoches CM, Campbell EG, Vogeli C, et al. Electronic health records' limited successes suggest more targeted uses. Health Aff (Millwood) 2010;29(4):639-646

24 Virapongse A, Bates DW, Shi P, et al. Electronic health records and malpractice claims in office practice. Arch Intern Med 2008;168 (21):2362-2367

25 Jones SC, McMenamin J, Kibbe DC. The interoperable electronic health record: preserving its promise by recognizing and limiting physician liability. Food Drug Law J 2008;63(1):75-87 